www.higieneanimal.ufc.br

\title{
La presencia de campylobacter en pollos de carne alimentados con diferentes niveles de butirato sódico en dietas experimentales
}

\author{
*WS, Benevides ${ }^{\text {*; MP, Díaz }}{ }^{2}$; VJ, Vinatea ${ }^{3}$; JM, Fragoso ${ }^{4}$ \\ ${ }^{1}$ Faculdade de Veterinaria, Universidade Estadual do Ceará, Fortaleza, Ceará, Brasil; \\ ${ }^{2}$ Departamento de Medicina y Cirugía Animal, Facultad de Veterinaria, Universidad Complutense de \\ Madrid, Madrid, Madrid, España; \\ 32Departamento de Producción Animal, Escuela de Ingenieros Agrónomos, Universidad Politécnica de \\ Madrid, Madrid, Madrid, España; \\ ${ }^{4}$ Servicio Técnico COBB Española, S.L., Madrid, España.
}

Summary: Comparisons of the effect for five different levels of butyrate were carried out, with or without the associated minero-vitamins mixture supplemented with essential amino acids and additives in the starter feed for phase I, 1-15 days old, resulting in ten treatments in total. In the diets for phase II, 1636 days old, there was no feed mixture and just one level of SB in the treatments for samples 2 to 5 and 7 to 10 . The samples 1 and 6 were the control group without SB. For this work we used a total of 960 oneday old broilers (Cobb-500), obtained from a commercial hatchery. The broilers were distributed into ten experimental treatments, with 8 repetitions per cage per treatment and 12 birds per cage, with a total of 80 cages and 960 broilers. At the end of the experiment after 36 days, a swab was collected from six birds per cage to assess the presence of campylobacter, but not positive results for this microorganism.

Autor para correspondencia. E.Mail: wsbvet@hotmail.com

Recebido em 20.04.2011. Aceito 20.07.2011 


\section{Introducción}

Según Torres \& Zarazaga (2002), Las sustancias antimicrobianas se emplean en veterinaria con fines terapéuticos y profilácticos para tratar o bien prevenir infecciones. En ambos casos, los antibióticos deben ser suministrados bajo el control de un profesional veterinario y la normativa vigente exige la prescripción de la receta veterinaria. El aislamiento de Campylobacter en granjas de Broilers es muy frecuente, considerándose comun a partir de cierta edad de los animales.

Chaveerach et al., (2004) no observaron diferencias significativas en las lesiones epiteliales del intestino delgado entre los grupos estudiados, empleando acido orgánico en el agua junto con una inoculación oral de Campylobacter $s p$ en pollos. Según Giacoboni et al., (1999), las especies Campylobacter jejuni y Campylobacter coli son agentes causales de enteritis en el hombre tanto en países en vías de desarrollo como en países desarrollados. Se estima que mas del $50 \%$ de los casos esporádicos de enteritis por Campylobacter estan asociados al consumo de pollos o a su manipulación.

\section{Materials and Methods}

El desarrollo experimental y los análisis quimicos se han realizado en las instalaciones del Departamento de Producción Animal de la Escuela Tecnica Superior de Ingenieros
Agrónomos de la Universidad Politecnica de Madrid.

Trabajamos con un total de 960 pollitos (Cobb- 500) de 1 dia de edad, procedentes de una incubadora comercial. Los pollitos fueran pesados individualmente y repartidos en un diseño experimental de bloques por peso a su llegada.

Se distribuyeron en diez tratamientos experimentales (TMT), con 8 replicas/jaulas por tratamiento y 12 pollos por jaula, con un total de 80 jaulas y 960 pollitos. Los animales se distribuyeron en diez tratamientos experimentales (TMT), con 8 replicas/jaulas por tratamiento y 12 pollos por jaula, con un total de 80 jaulas y 960 pollitos (Tabla 01). El agua fue ofrecida ad libitum y las dietas fueron formuladas isoproteicas e isocaloricas de acuerdo con las recomendaciones de NATIONAL RESEARCH CONCIL (1994).

El diseño factorial (tabla 01) fue compuesto de 2 piensos con 5 niveles de butirato sódico (BS). Los TMT/piensos fueran suplementados con BS en diferentes niveles (\% BS/piensos) solamente en la primera edad (pienso de arranque).

Los tratamientos 7 a 10 tuvieron añadido un núcleo o corrector comercial con una incorporación de minerales y aminoacidos esenciales (AAe). 
Tabla 1. Compuesto de 2 piensos con 5 niveles de butirato sódico (BS).

\begin{tabular}{|l|c|c|c|c|c|}
\hline & \multicolumn{5}{|c|}{$\%$ BS / PIENSOS } \\
\cline { 2 - 6 } & 0 & $0,15 \%$ & $0,30 \%$ & $0,45 \%$ & $0,60 \%$ \\
\hline $\begin{array}{l}\text { Pienso I } \\
\text { (sin NúcLEO AAe) }\end{array}$ & $\mathrm{T} 1$ & $\mathrm{~T} 2$ & $\mathrm{~T} 3$ & $\mathrm{~T} 4$ & $\mathrm{~T} 5$ \\
\hline $\begin{array}{l}\text { Pienso II } \\
\text { (con NúCLEO AAe) }\end{array}$ & $\mathrm{T} 6$ & $\mathrm{~T} 7$ & $\mathrm{~T} 8$ & $\mathrm{~T} 9$ & $\mathrm{~T} 10$ \\
\hline
\end{tabular}

Al final del experimento con 36 días, se tomó un hisopo de un pollo por jaula para evaluar la presencia de Campylobacter, los cuales debidamente identificados, fueran enviados al Laboratorio Nacional de Sanidad Animal de Algete (Madrid).

\section{Resultados y Discusión}

Los hisopos tomados para evaluar la presencia de Campylobacter sp., no presentaron resultados positivos. Por tanto, la ausencia de esta bacteria en todos los lotes de animales, no permite detectar ningún efecto del BS en condiciones experimentales.

Tampoco Chaveerach et al., (2004), no observaron diferencias significativas en el intestino delgado entre los grupos estudiados, administrando un ácido orgánico al agua juntamente con una inoculación oral de Campylobacter sp. en pollos de carne.

Como se ha indicado, Salazar et al. (2008) concluyeron que la inconsistencia de respuestas y diferencias puede ser debido ala falta de desafíos sanitarios en condiciones experimentales; por lo que posiblemente en condiciones de granja, los efectos del BS podrían verse con mayor diferencia.

\section{Conclusión}

Los resultados microbiológicos de los hisopos tomados de la cloaca de los pollos a los 36 días, no dieron lugar a resultados positivos a la presencia de Campylobacter en ninguna de las jaulas, por lo que no podemos considerar que los tratamientos estudiados tengan efecto en las condiciones experimentales.

\section{Referências Bibliográficas}

CHAVEERACH, P., KEUZENKAMP, D. A., LIPMAN, L. J. A. AND VAN KNAPENT, F. (2004). Effect of Organic acids in drinking water for young broilers on Campylobacter infection, Volatile Fatty Acid

Production, Gut Microflora And histological cell changes. Poultry Science, 83: 330-334.

GIACOBONI, G., PUCHURI, M.C. Y CERDÁ R. C. 1999). Campylobacter termotolerantes en menudos y carcasas de pollos provenientes de diferentes comercios 
de la Ciudad de la Plata (Argentina).

Analecta Veterinaria 19, 1: 51-54.

NATIONAL RESEARCH COUNCIL (1994).

Nutrient Requirements of Poultry, 9th ed.

.SALAZAR, P.C. R., ALBUQUERQUE, R.,

TAKEARA, P., TRINDADE NETO, M.A.E

FRANCELINO, L. (2008). Efeito dos ácidos

lático e butírico, isolados e associados, sobre o

desempenho, imunidade humoral e morfometria intestinal em frangos de corte. Brazilian Journal Veterinarian Research Animal Science, São

Paulo, 45(6): 463 - 471.

TORRES C.Y ZARAZAGA M. (2002)

Antibióticos como promotores del crecimiento en animales. ¿Vamos por el buen camino? Departamento de Agricultura y Alimentación. Universidad de La Rioja, Logroño. Gaceta Sanitaria. 16(2):109-112.
National Academy of Science, Washington, DC. 176pp. 
Benevides et al., Revista Brasileira de Higiene e Sanidade Animal (v.5, n.2) p. 1-4, jul - dez (2007) 